\title{
TEMA 1-2014: GENERALIDADES SOBRE SÍNDROME GOMPARTIMENTAL EN EXTREMIDADES
}

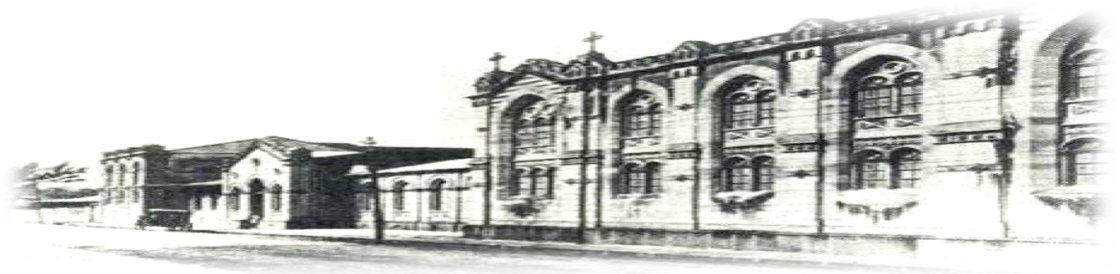

Hospital San quan de Dias, San José, Costa Rica. Fundada en 1845

Recibido: $\quad 13 / 10 / 2013$

Aceptado: $\quad 23 / 11 / 2013$

Daniela Jiménez Soto ${ }^{1}$

Javier Soto Fallas ${ }^{2}$

${ }^{\mathbf{1}}$ Médica general. Correo electrónico: danajs2509@hotmail.com

${ }^{2}$ Especialista en Ortopedia y Traumatología. Asistente del Servicio de Ortopedia. Sección de Cirugía. Hospital San Juan de Dios.

\section{RESUMEN}

El síndrome compartimental que no se reconoce y no se trata de manera inmediata puede llevar a la pérdida de la extremidad y en el peor de los casos amenaza la vida del paciente. El pronóstico para la recuperación depende del diagnóstico y tratamiento inmediato. El dar un diagnóstico tardío o equivocado aumenta el número de complicaciones y riesgos. Esta revisión de tema se basa en hacer énfasis en la clínica del paciente, sobre todo si el dolor en la extremidad es lo que predomina ante las medidas de presión compartimental que se puedan obtener. No hay un estándar de oro para el diagnóstico y la discrepancia entre la gravedad del dolor, comparada con el grado de trauma, por lo tanto el diagnóstico puede ser insidioso en algunos casos, de modo que siempre se debe mantener alto nivel de sospecha.

\section{PALABRAS CLAVE}

Síndrome Compartimental. Manifestaciones clínicas. Dolor e Isquemia.

\begin{abstract}
Compartment syndrome is not recognized and is not immediately can lead to loss of the limb and in the worst cases life-threatening. The prognosis for recovery depends on the diagnosis increases the number of complications and risks. This review of topics is based on making emphasis on the patient's clinical pain especially if the tip is what prevails before compartmental pressure measurements to be obtained. There is no gold standard with the degree of trauma so the diagnosis can be insidious in some cases, therefore you should always keep the suspicion.
\end{abstract}

\section{KEY WORDS}

Compartment syndrome. Clinical Picture. Ischemic pain.

\section{INTRODUCCIÓN}

Se ha pensado durante más de un siglo que un aumento en la presión de un compartimiento era 
debido a un mecanismo central, así lo planteaba Von Volkmann en sus primeros trabajos sobre síndrome compartimental ${ }^{(5)}$. Estos principios e ideas se han ido refinando gracias al aumento de conocimientos de la anatomía y la evolución de las técnicas de diagnóstico.

Dentro de las extremidades hay músculos que están organizados en compartimientos apretados. Cada músculo contiene arterias, venas, nervios y vasos linfáticos rodeados de un tejido conectivo elástico conocido como vainas. Estas vainas limitan y acomodan el volumen del contenido y dividen a las extremidades en compartimientos, por lo tanto, un aumento de la presión dentro de un compartimiento muscular hasta el punto donde se reduce o elimina la perfusión vascular necesaria para la viabilidad de los tejidos allí comprendidos, es conocido como síndrome compartimental; donde puede haber un aumento del contenido o disminución del continente ${ }^{(5,8)}$.

Pueden ser agudos o crónicos y localizados o generalizados (estos últimos tienen expresión sistémica: síndrome de aplastamiento o crush syndrome).

\section{DISCUSIÓN}

\section{Síndrome Compartimental Agudo (SCA)}

\section{Etiología}

Las causas del síndrome compartimental se dividen en 2 grandes grupos, de acuerdo Matsen y Rowland $^{(6)}$. Se hará referencia únicamente a las más frecuentes:

\section{Por aumento de la presión intracom- partimental (aumento del contenido):}

En el 69\% de los casos se asocia a traumatismo directo de alta energía, sobre todo fracturas cerradas de huesos largos, aunque también puede darse en abiertas ${ }^{(2,5)}$. En los adultos las más frecuentes $(40 \%)$ son a nivel de diáfisis tibial y también se dice que las fracturas de meseta tibial dan lugar a un SCA en un $30 \%$, de predominio Schatzker tipo IV y supracondíleas de húmero (en niños) ${ }^{(1,2,6)}$.

El edema intersticial, el hematoma secundario, la lesión arterial y de partes blandas son los causantes del aumento de presión en un compartimiento con volumen constante ${ }^{(1)}$.

\section{Por disminución del volumen compar- timental (disminución del continente):}

En quemaduras o congelaciones con escara circular, aplastamiento por un objeto pesado durante horas, cierres quirúrgicos, vendaje muy constreñidos y mordeduras de serpiente ${ }^{(3,5,6)}$. En todos estos casos se altera la vascularización de los tejidos incluso sin existir lesión vascular.

\section{Fisiopatología}

Bien por el aumento del contenido o por disminución del continente, existe un problema común: el aumento de presión en un compartimiento. Se explica con la teoría de gradientes arteriovenosos de Matsen y Rorabeck, en la que el flujo sanguíneo local en un compartimiento es igual a la presión arterial local menos la presión venosa local, dividido entre la resistencia vascular local.

Debido a este aumento de la presión, la circulación capilar se enlentece o se anula, provocando isquemia muscular y lesiones musculares permanentes. La circulación capilar es una circulación de baja presión; por eso aumentos en la presión del compartimiento de tan solo $30 \mathrm{mmHg}$ pueden provocar el síndrome. Al ocurrir la lesión, se disminuye el aporte sanguíneo al compartimento. La pobre perfusión celular produce una disminución de los depósitos intracelulares de energía para mantener el metabolismo normal y se utiliza el metabolismo anaerobio, el cual no es capaz de suministrar las cantidades necesarias y adecuadas de ATP, produciendo como evento final acumulación de ácido láctico ${ }^{(6)}$.

La falta de ATP ocasiona un trastorno a nivel de la membrana celular y en la bomba de $\mathrm{Na}^{+} / \mathrm{K}^{+}$, produciendo un aumento de la permeabilidad capilar, lo que permite la salida de $\mathrm{K}^{+}$y la entrada de $\mathrm{Na}^{+}, \mathrm{Ca}^{++}$y agua al espacio extracelular, originando edema intersticial y elevación de la presión intracompartimental. La entrada de $\mathrm{Ca}^{++}$lesiona las mitocondrias, donde se almacena la energía en forma de ATP. Lo anterior trae como consecuencia que las pequeñas venas y capilares se ocluyan, agravando la hipoxia ya existente. 
Luego sucede un fenómeno compensatorio de reperfusión, durante el cual el oxígeno penetra la célula, pero esto lejos de ayudar, agrava el fenómeno, ya que se liberan radicales libres que producen mayor daño tisular al activar y liberar las proteasas y fosfolipasas; se aumenta la agregación plaquetaria y se produce coagulación intravascular de la microcirculación, iniciándose la lisis celular con daño muscular y nervioso ${ }^{(6)}$.

Algunos estudios de Whiteside y Heckmann han demostrado que a las cuatro horas de isquemia es cuando empiezan a verse cambios irreversibles en el músculo esquelético y alteraciones en la conducción nerviosa de tipo neuroapraxia. A las ocho horas existe ya axonotmesis y destrucción celular, ambos irreparables.

Para que no se palpen los pulsos distales es necesario que la presión del compartimiento iguale o bien sobrepase la presión sistólica (150 $\mathrm{mmHg}$ ). Por eso en muchos síndromes compartimentales, los pulsos distales están conservados.

En general, estos cambios aparecen cuando la presión intracompartimental supera los 30 mmHg y se agrava según el tiempo de evolución y según susceptibilidad individual.

\section{Historia clínica}

La historia precisa de un paciente con sospecha de SCA es de suma importancia, sin embargo no siempre es posible obtenerla de manera confiable o clara. Ejemplos reales de estos casos son los pacientes comatosos politraumatizados, pacientes intoxicados y niños pequeños. En estos casos el diagnóstico se hace por medida de presiones.

Cuando la información puede ser recuperada se debe tener especial cuidado en los datos sobre el tipo de lesión, el grado de dolor y el tiempo. Estas medidas proporcionan un importante apoyo para realizar el diagnóstico ${ }^{(1-2,6)}$. Se debe ser enfático en el principal síntoma, que es el dolor desproporcionado a la intensidad del traumatismo. El paciente se muestra cada vez más inquieto por el aumento del dolor. Tras varias horas este empieza a disminuir (debido a la isquemia irreversible de los tejidos).

\section{Examen Físico}

Tras la inspección, el hallazgo principal es la inflamación de la extremidad. La incapacidad de poder movilizar la extremidad afectada, la palidez, ausencia de pulsos (no siempre), las parestesias y parálisis pueden ser otros síntomas indicadores $^{(1,6)}$.

El diagnóstico precoz es lo más importante, por eso el dolor desproporcionado que aumenta con la movilización pasiva de los músculos y que con curso progresivo debe ser de sospecha mayor. En cuanto a la ausencia de los pulsos, desde la llegada del paciente a urgencias de debe descartar otro tipo de lesión vascular (mediante arteriografía, principalmente), puesto que en el SCA es de aparición más tardía. Esto ocurre también con el déficit motor.

\section{Diagnóstico}

Es muy importante la exploración seriada del paciente porque así es como se detecta el aumento progresivo del dolor, el dato clínico más importante.

Algunos datos de laboratorio y gabinete pueden ayudar:

- Aumento de la creatinfosfocinasa (CPK) en el plasma, aunque resulta tardía por ser un signo ya de necrosis.

- Aumento de la creatinina y el nitrógeno ureico (indican afección renal asociada).

- La mioglobina en orina confirma la destrucción de los miocitos.

- El eco-doppler y la arteriografía nos sirven para descartar otras patologías vasculares.

La prueba complementaria más útil parece ser la medición de la presión intracompartimental.

\section{Basándose en la presión arterial diastó- lica.}

Hay autores que utilizan la presión diastólica como referencia para definir el umbral a partir del cual es necesario descomprimir un compartimiento, porque la perfusión tisular depende de la presión diastólica. En este caso indica entonces una fasciotomía cuando la diferencia entre la presión diastólica y la intracompartimental es inferior a $30 \mathrm{mmHg}$

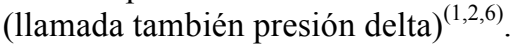


Ejemplo: una presión diastólica de $70 \mathrm{mmHg}$ y una presión del compartimiento de $45 \mathrm{mmHg}$ indicarían fasciotomía (diferencia de $25 \mathrm{mmHg}$ ), sin embargo, $35 \mathrm{~mm} \mathrm{Hg}$ no (diferencia de 35 $\mathrm{mmHg}$ ).

\section{Basándose en valores absolutos:}

Otros autores definen valores absolutos en el compartimiento, indicando observación si la presión es menor de $30 \mathrm{mmHg}$ y fasciotomía si es mayor a $40 \mathrm{mmHg}$. En valores entre los 30 y 40 la indicación dependerá de la sospecha clínica.

Como ya se dijo, existen variaciones individuales en la tolerancia a la isquemia y la medida de la presión intracompartimental no debe ser el único dato en que se base la indicación quirúrgica.

\section{Tratamiento}

El SCA es una emergencia quirúrgica y debe ser tratada de forma inmediata ${ }^{(1)}$. El tratamiento consiste en liberar el compartimiento afectado mediante la apertura de la fascia que la envuelve

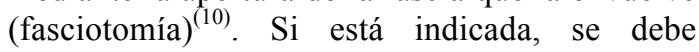
realizar con urgencia, antes incluso que la limpieza de heridas o la inmovilización del foco de la fractura.

Se realiza con una incisión en la piel en casi toda la longitud del miembro y se abre la fascia a través de ella. No debe hacerse de manera subcutánea porque esto no asegura una descompresión adecuada. Una vez hecha, hay que comprobar que los músculos afectados se encuentran totalmente liberados. Normalmente, se debe evitar cualquier tipo de debridamiento o resección de tejido necrótico en ese primer acto quirúrgico para prevenir más traumatismo a la zona.

\section{Localización de las incisiones ${ }^{(5)}$}

- Zona glútea: incisión posterolateral para descomprimir glúteo mayor, mediano y menor.

- Muslo: incisión sobre los aductores, cuádriceps, y/o isquiotibiales dependiendo de los compartimientos afectados.

- Pierna: presenta cuatro compartimientos: anterior, peroneo (lateral), posterior profundo y posterior superficial; se pueden abrir con dos únicas incisiones: una lateral para acce- der a los dos primeros y una interna para los dos posteriores.

- Pie: tiene nueve compartimientos: medial, aductor, calcáneo, superficial y lateral (que se abren mediante una incisión interna) y cuatro interóseos (que precisa dos incisiones dorsales).

- Brazo: dos compartimientos y dos incisiones necesarias: anteromedial y posterior.

- Antebrazo: tres compartimientos: volar (palmar, anterior y flexores-pronadores), dorsal (posterior de los músculos extensoressupinadores) y el radial (primer y segundo radial y supinador largo). Los tres están comunicados (los envuelve una única fascia), por lo que suele ser suficiente con una incisión palmar que llega hasta el túnel del carpo.

- Mano: los interóseos se liberan por dos incisiones dorsales y los palmares mediante incisiones sobre eminencias tenar e hipotenar.

Las heridas se cubren con compresas húmedas y se aplica un vendaje levemente compresivo. En posteriores intervenciones se debe realizar un lavado profuso de los compartimientos abiertos y resección de las zonas no viables del músculo. Se debe repetir tantas veces como sea necesario, hasta garantizar la estabilidad y viabilidad de los tejidos.

Cuando el SCA ya está establecido y se observa isquemia completa e irreversible con sus consiguientes deformaciones (síndrome de contractura isquémica de Volkmann), es preferible no abrir el compartimiento muscular, porque esto no genera ningún beneficio (no se recuperan las funciones cuando ha llegado a este punto). Además puede propiciar la aparición del síndrome de aplastamiento, debido a la liberación de toxinas y detritus celulares al torrente sanguíneo.

\section{Complicaciones}

La secuela más frecuente es una deformidad permanente en el miembro afectado: La contractura de Volkmann se produce por una isquemia prolongada en un compartimiento que provoca necrosis de estructuras musculares y nerviosas. En estos casos las fibras musculares se sustituyen progresivamente por fibrosis y van creándose adherencias y contracturas, con deformidades muy típicas según los compartimientos afectados: 
- Miembro superior: flexión y pronación del codo con flexión de la muñeca. En la mano, extensión de articulaciones metacarpofalángica, flexión de las interfalángicas y aducción del pulgar (mano en garra).

- Miembro inferior: más variable. Puede haber pie equino o equinovaro cavo, metatarso varo o dedos en garra.

En casos leves se intenta corregir la deformidad con férulas, pero en casos graves son necesarias varias fases: liberación de nervios afectados (hasta el año de la lesión puede existir recuperación neurológica parcial), resección de zonas infartadas, tenotomías, alargamiento, transposiciones tendinosas, injertos nerviosos o musculares vascularizados, osteotomías etc.

En último lugar pueden ser necesarias las artrodesis, resección del carpo proximal, o incluso la amputación si el dolor es insoportable o existen úlceras o deformidades intratables. El objetivo principal es recuperar la funcionalidad del miembro pero en ocasiones no es posible

\section{Síndrome de aplastamiento (crush syndrome)}

Consiste en cambios sistémicos tras la revascularización de un compartimiento necrótico. Se deben a la liberación de toxinas intracelulares y a la presencia de mioglobina en plasma $^{(11)}$.

\section{Consecuencias}

- Acidosis sistémica e hipercalemia: arritmia cardiaca.

- Extravasación de fluidos: choque hipovolémico.

- Mioglobinemia: fallo renal.

Todo esto provoca un fracaso multiorgánico que precisa ingreso a la UCI, para monitorización y vigilancia intensiva, reposición de fluidos e intubación o hemodiálisis en algunos casos.

\section{Síndrome Compartimental Crónico}

Horn fue el primero en describir el síndrome compartimental crónico a la que le llamó gangrena de la marcha tras un esfuerzo prolongado $^{(4,6)}$. Se diferencia de los agudos en que aparecen sin antecedentes traumáticos, puede reproducirse regularmente $y$ no requieren tratamiento urgente.

\section{Fisiopatología}

Se han descrito los siguientes factores predisponentes:

- Fascia del compartimiento muy rígida.

- Aumento del volumen muscular por hipertrofia del mismo, edema o incremento de la permeabilidad capilar.

- Flujo linfático o venoso restringido.

- Hemorragia por la rotura de fibras musculares.

No se ha podido demostrar que la elevación de la presión intracompartimental durante el ejercicio disminuya la perfusión a los tejidos, ni que sea este hecho el que provoque el dolor que los pacientes relatan. Se han barajado otras causas como la irritación del periostio o la estimulación de los receptores musculares de presión para explicar este dolor.

\section{Cuadro Clínico}

En varones jóvenes, deportistas y soldados generalmente en miembros inferiores, aparece dolor, calambres, quemazón. En ocasiones debilidad y parestesias localizadas en el compartimiento afectado. También refieren fatiga muscular prematura y mejoría de los síntomas al interrumpir el ejercicio ${ }^{(4)}$.

$\mathrm{Su}$ clínica confusa puede hacer pensar en fracturas por estrés, microdesgarros musculares (agujetas) y periostitis interna de la tibia. Se distingue porque el SCC no tiene gammagrafía positiva, ni pico de dolor a las 48 horas, ni dolor a la palpación, como tendrán las patologías mencionadas anteriormente.

\section{Diagnóstico}

Exploración física anodinia en reposo. Suele ser bilateral $(75-95 \%$ casos $)$, pero con clínica más evidente en uno de los miembros. Tras el ejercicio se puede observar aumento de consistencia del compartimento, con dolor a la palpación y a veces hernias musculares.

La radiografía y gammagrafía normales, solo sirven para descartar fracturas por estrés o reacciones periósticas. En los pacientes con 
historia y exploración física compatibles con síndrome compartimental crónico por ejercicio, al diagnóstico certero se llega mediante la medición de la presión intracompartimental inmediata antes y después del esfuerzo. Los criterios aceptados en general, son una presión en reposo mayor a $15 \mathrm{mmHg}$, una presión un minuto después del ejercicio mayor de $30 \mathrm{mmHg}$, o una presión a los cinco minutos de $20 \mathrm{mmHg}$.

\section{Tratamiento}

El tratamiento conservador consiste en intentar modificar la actividad del paciente, para no provocar los síntomas si falla. En cuanto al quirúrgico, es la única opción de tratamiento efectiva tras el diagnóstico de certeza de un síndrome compartimental crónico. Puede realizarse una fasciotomía subcutánea o una fasciectomía del compartimiento.

En la pierna, mediante dos incisiones anterolaterales de unos $2 \mathrm{~cm}$ de longitud para los compartimientos anterior y peroneo y otras dos mediales para los posteriores. Esto mejora generalmente la clínica. Se debe garantizar la descompresión de los compartimientos afectados y dejar un drenaje subcutáneo para evitar la aparición de un SCA. Colocar un vendaje levemente compresivo y frío local. Reposo de dos semanas y reinicio de las actividades deportivas las 4 a 6 semanas.

\section{CONCLUSIONES}

La tardanza en el reconocimiento de SCA en la gran mayoría de los casos trae resultados adversos y en los casos no reconocidos pueden dejar a los pacientes con extremidades no viables que requieran de amputación o mortalidad.

Se dice que la tasa de mortalidad en pacientes con SCA es de un $47 \%$ debido a diagnóstico retrasado.

Se debe insistir en los signos, síntomas, sospecha y sobre todo que se detecte de manera precoz para evitar todas sus consecuencias. Tomando en cuenta que la gran mayoría de los pacientes con este tipo de afección son hombres jóvenes con vida activa y la prioridad es introducirlos a la vida cotidiana sin ningún tipo de consecuencia que vaya a ser traumática o limitante para el paciente.

\section{REFERENCIAS BIBLIOGRÁFICAS}

1. Oprel PP Eversdijk MJ Vlot J Tuinebreijer WE den Hartog D. The Acute Compartment Syndrome of the Lower Leg: A Difficult Diagnosis? The Open Orthop J. 2010;4:115119.

2. Mc Queen MM Gaston P Court-Brown CM. Acute Compartment Syndrome. Who is at risk? J Bone Joint Surg Br. 2000;82(2):200-203.

3. Cortes S Pacheco EM Martel D Millán AM García M Spreafico M Ginovés M. Síndrome Compartimental Bilateral en Miembros Inferiores tras Cirugía de Schwannoma Lumbar en Posición Genupectoral. Servicio de Neurocirugía. Unidad de Recuperacón Postanestésica Universitaria de Canarias, La Laguna. Santa Cruz de Tenerife. 2007;18(1):56-59.

4. García S Hidalgo A Martínez M. Síndrome Compartimental Crónico de Esfuerzo. Afectación Bilateral del Compartimiento Anteroexterno de la Pierna en Deportistas. Rev Esp Cir Osteoart. 1996;31:299-304.

5. Tapiwa M Malahias M Hindocha S Khan W Juma A. Acute Compartment Syndrome of the Limbs: Current Concepts and Management. The Open Orthop J. 2012;6:535543.

6. Patiño Bravo Arturo. Síndrome Compartimental. Fundación Sudamericana de Cirugía de La Mano. 2012;1-13.

7. Power RA Greengross P. Acute Lower Leg Compatment Syndrome. The Journal of Bones and Joint Surgery. 1991;25:218-220.

8. Tuckey J. Bilateral Compartment Syndrome Complicating Prolonged Lithotomy Position. Case Report. British Journal of Anaesthesia. 1996;77:546-549.

9. Harvey EJ Sanders DW Shuler MS. What's New in Acute Compartment Syndrome? The J Orthop Trauma. 2012;26(12):699-702.

10. Gómez JC Hernando C. Fasciotomía Profiláctica y Síndrome Compartimental de las Extremidades: ¿Existen indicaciones justificables? Rev Colomb Cir. 2011;26:101110.

11. Duckworth A McQueen M. Diagnosis of Acute Compartment Syndrome. J Bone Joint Surg Am. 2011;51:1-8.

12. Shadgan B Menon M Berry G Martin C Duffy P Syephen D. Current Thinking about Acute Compartment Syndrome of the Lower Extremity. Annual CJS/COA Sym- 
posium. Canadian Medical Association. 2010;329-334.

13. Rowland SA. Faciotomy: The Treatment of Compartment Syndrome. Greens Operative Hand Surgery, $4^{\text {th }}$ ed, New York.1999;689710.

14. Rekha A. Compartment Syndrome. Academic Journal. 2010;2(2):28-30.

15. Battacharyya $\mathrm{T}$ Vrahas $\mathrm{M}$. The Medical Legal Aspects of Compartment Syndrome. J Bone and Joint Surg Am. 2004;86:864-868.

16. Wall C Richardson $\mathrm{M}$ Destiger $\mathrm{R}$ et al. Compartment Syndrome. Trauma Service Guidelines. The Royal Melbourne Hospital. 2012;1-5

DECLARACIÓN DE CONFLICTO DE INTERESES

Los autores declaran que no existen conflictos de intereses. 